\title{
Characterization of anticoagulant heparinoids by immunoprofiling
}

\author{
Tessa J. Wijnhoven • Els M. van de Westerlo • \\ Nicole C. Smits • Joost F. Lensen • Angelique L. Rops • \\ Johan van der Vlag • Jo H. Berden • \\ Lambert P. van den Heuvel • Toin H. van Kuppevelt
}

Received: 22 May 2007 /Revised: 20 August 2007 / Accepted: 28 August 2007 / Published online: 2 October 2007

(C) Springer Science + Business Media, LLC 2007

\begin{abstract}
Heparinoids are used in the clinic as anticoagulants. A specific pentasaccharide in heparinoids activates antithrombin III, resulting in inactivation of factor Xa andwhen additional saccharides are present-inactivation of factor IIa. Structural and functional analysis of the heterogeneous heparinoids generally requires advanced equipment, is time consuming, and needs (extensive) sample preparation. In this study, a novel and fast method for the characterization of heparinoids is introduced based on reactivity with nine unique anti-heparin antibodies. Eight heparinoids were biochemically analyzed by electrophoresis and their reactivity with domain-specific anti-heparin antibodies was established by ELISA. Each heparinoid displayed a distinct immunoprofile matching its structural characteristics. The immunoprofile could also be linked to biological characteristics, such as the anti-Xa/anti-IIa ratio, which was reflected by reactivity of the heparinoids with antibodies HS4C3 (indicative for 3-O-sulfates) and HS4E4 (indicative for domains allowing anti-factor IIa activity). In addition, the immunoprofile could be indicative for heparinoid-induced
\end{abstract}

T. J. Wijnhoven • E. M. van de Westerlo N. C. Smits •

J. F. Lensen $\cdot$ T. H. van Kuppevelt $(\triangle)$

Department of Matrix Biochemistry,

Nijmegen Centre for Molecular Life Sciences,

P.O. Box 9101, 6500 HB Nijmegen, The Netherlands

e-mail: A.vankuppevelt@ncmls.ru.nl

T. J. Wijnhoven · L. P. van den Heuvel

Department of Pediatric Nephrology,

Radboud University Nijmegen Medical Centre,

P.O. Box 9101, 6500 HB Nijmegen, The Netherlands

\section{A. L. Rops $\cdot$ J. van der Vlag $\cdot$ J. H. Berden}

Nephrology Research Laboratory, Division of Nephrology,

Nijmegen Centre for Molecular Life Sciences,

Radboud University Nijmegen Medical Centre,

P.O. Box 9101, 6500 HB Nijmegen, The Netherlands side-effects, such as heparin-induced thrombocytopenia, as illustrated by reactivity with antibody NS4F5, which defines a very high sulfated domain. In conclusion, immunoprofiling provides a novel, fast, and simple methodology for the characterization of heparinoids, and allows high-throughput screening of (new) heparinoids for defined structural and biological characteristics.

Keywords Antibody Anticoagulation .

Heparin-induced thrombocytopenia $\cdot$ Heparinoid .

Immunoprofiling

\section{Introduction}

Glycosaminoglycans (GAGs) are long, unbranched, negatively charged polysaccharide chains. The precursor of heparin and heparan sulfate (HS) is composed of alternating $\mathrm{N}$-acetylglucosamine (GlcNAc) and D-glucuronic acid (GlcA) residues. In chondroitin sulfate (CS) and dermatan sulfate (DS), the aminosugar is a $N$-acetylgalactosamine (GalNAc) instead of GlcNAc. The polysaccharide chain precursors are modified by a sequential series of enzymatic reactions, including $N$-deacetylation $/ N$-sulfation, C5epimerization of GlcA to L-iduronic acid (IdoA), and $O$ sulfation at various positions [27, 32].

Heparin is widely used as an anticoagulant. A specific pentasaccharide sequence (GlcNAc/NS(6OS)-GlcA$\operatorname{GlcN} S(3,6 \mathrm{OS})-\operatorname{IdoA}(2 \mathrm{O} S)-\mathrm{GlcN} S(6 \mathrm{O} S))[5,23,37]$ in heparin binds with high affinity to the enzyme inhibitor antithrombin III, causing a conformational change, resulting in the exposition of its active sites and in inactivation of factor Xa. For the inactivation of factor IIa, an additional site in heparin proximal to the pentasaccharide is necessary. The formation of a ternary complex between antithrombin 
III, factor IIa and heparin, which requires at least 18 saccharide residues for efficient formation, subsequently results in the inactivation of factor IIa [23]. Next to heparin, other heparinoids are used, including preparations containing other GAGs such as HS and DS, low molecular weight heparins (LMWHs), and fondaparinux, a synthetic pentasaccharide identical in structure to the antithrombin IIIbinding pentasaccharide. Heparinoids are used to treat patients with venous thrombosis and pulmonary embolism $[2,5,10,22]$. In addition, they are used to prevent thrombosis in patients that undergo surgery and to prevent clotting in the extracorporeal circulation during hemodialysis $[2,5,10,22,24,28]$. Due to their lower molecular weight, LMWHs have a high anti-factor Xa activity compared to their anti-factor IIa activity resulting in a more refined regulation of coagulation $[4,24]$. In addition, the biological half-life of LMWHs/fondaparinux is longer in comparison to heparin, and the incidence of heparininduced thrombocytopenia (HIT) is substantially reduced $[6,7,23]$.

LMWHs are synthesized from heparin by controlled chemical or enzymatic depolymerization processes, resulting in the production of oligosaccharides of various lengths (Table 1). Dalteparin and nadroparin are produced by deamination using nitrous acid, resulting in the formation of an unnatural 2,5-anhydromannitol residue at the reducing end of the resulting oligosaccharides. Enoxaparin is produced by chemical $\beta$-elimination at alkaline $\mathrm{pH}$, whereas tinzaparin is produced by enzymatic $\beta$-elimination by heparinase. Both processes result in the formation of an unsaturated uronic acid residue at the non-reducing end of the GAG fragments $[12,17]$.

All heparinoids, with the exception of fondaparinux, are heterogeneous mixtures of different GAG chains. Structural analysis of heparinoids is generally done by nuclear magnetic resonance spectroscopy, high pressure liquid chromatography, mass spectrometry, capillary electrophoresis, or polyacrylamide gel electrophoresis [17]. In general, these techniques require sophisticated instrumentation and sometimes extensive sample preparation. Alternative methodologies that are fast and simple are needed. Immunoprofiling of heparinoids could be a fast and simple method to characterize the complex heparinoids. Recently, a number of phage-display-derived antibodies were generated, reactive with various domain structures within heparin. In this study nine antibodies-all defining different heparin epitopes-were applied to study their reactivity with heparinoids.

\section{Materials and methods}

\section{Heparinoids}

The following heparinoids were included in the study: heparin (Sigma, St. Louis, MO), dalteparin (Pharmacia, Woerden, The Netherlands), nadroparin (Sanofi-Synthélabo, Maassluis, The Netherlands), enoxaparin (Aventis Pharma, Hoevelaken, The Netherlands), tinzaparin (LEO Pharma, Breda, The Netherlands), sulodexide (Alfa Wassermann S.p.A., Bologna, Italy), danaparoid (Organon, Oss, The Netherlands), and fondaparinux (Sanofi-Synthélabo, Maassluis, The Netherlands). Characteristics are given in Table 1.

\section{Quantification of glycosaminoglycans by Farndale assay}

A Farndale assay was done to determine the concentration of GAGs in the preparations [13]. To $50 \mu \mathrm{l}$ of the standard (heparin, $0-100 \mu \mathrm{g} / \mathrm{ml}$ ) and heparinoids $1 \mathrm{ml}$ Farndale reagent $(40.5 \mathrm{mmol} / \mathrm{l}$ glycine, $40.6 \mathrm{mmol} / \mathrm{l} \mathrm{NaCl}$, and $0.05 \mathrm{mmol} / 1$ 1,9-dimethylmethylene blue, $\mathrm{pH}$ 3.0) was added and absorbance was measured directly at $525 \mathrm{~nm}$.

Table 1 Characteristics of therapeutic heparinoids

\begin{tabular}{llc}
\hline Heparinoid: generic name (brand name) & Composition (and method of heparin modification) & $\begin{array}{c}\text { Molecular } \\
\text { weight, kDa }\end{array}$ \\
\hline Heparin & Heparin & $5.0-30.0$ \\
Dalteparin (Fragmin) & LMWH (deaminative cleavage with nitrous acid) & $4.0-6.0$ \\
Nadroparin (Fraxiparine) & LMWH (deaminative cleavage with nitrous acid) & $2.4-7.2$ \\
Enoxaparin (Clexane, Lovenox) & LMWH ( $\beta$-eliminative cleavage by alkaline treatment) & $3.5-5.5$ \\
Tinzaparin (Innohep, Logiparin) & LMWH ( $\beta$-eliminative cleavage by heparinase) & $3.4-5.6$ \\
Sulodexide (Vessel Due F) & $80 \%$ 'fast-moving' heparin ${ }^{\mathrm{a}}, 20 \%$ DS & $<8.0$ \\
Danaparoid (Orgaran) & $84 \% \mathrm{HS}, 12 \%$ DS, $4 \% \mathrm{CS}$, & $4.0-10.0$ \\
Fondaparinux (Arixtra) & Pentasaccharide: $\mathrm{C}_{31} \mathrm{H}_{43} \mathrm{~N}_{3} \mathrm{Na}_{10} \mathrm{~S}_{8}$ & 1,728 \\
\hline
\end{tabular}

All drugs are derived from porcine intestinal mucosa, with the exception of the chemically synthesized pentasaccharide fondaparinux. $L M W H$ Low-molecular weight heparin, $H S$ heparan sulfate, $D S$ dermatan sulfate, $C S$ chondroitin sulfate

${ }^{a}$ Heparin that is less sulfated and has a lower molecular weight [42]. 
Determination of glycosaminoglycan classes by agarose gel electrophoresis

The different classes of GAGs in the heparinoids were separated by agarose gel electrophoresis, fixed in $0.1 \%$ $(w / v)$ azure $\mathrm{A}$ in $10 \mathrm{mmol} / 1 \mathrm{MgCl}_{2} \cdot 6 \mathrm{H}_{2} \mathrm{O}, 50 \mathrm{mmol} / \mathrm{l} \mathrm{Na}-$ formiate and $0.001 \%(w / v) \mathrm{Na}$-azide $(\mathrm{pH} 3.5)$, and stained by combined azure A-silver treatment [38].

\section{Enzymatic digestion of heparinoids}

Heparinoids were diluted in heparinase I digestion buffer $\left(50 \mathrm{mmol} / 1 \mathrm{NaAc}\right.$ and $50 \mathrm{mmol} / 1 \mathrm{Ca}(\mathrm{Ac})_{2}$, $\mathrm{pH}$ 7.0) and/or chondroitinase $\mathrm{ABC}$ digestion buffer ( $25 \mathrm{mmol} / \mathrm{l}$ Tris and $2 \mathrm{mmol} / \mathrm{l} \mathrm{Mg}(\mathrm{Ac})_{2}, \mathrm{pH} \mathrm{8.0)}$ to a final content of $4 \mathrm{mg} / \mathrm{ml}$. The enzymes were added to a final concentration of $0.02 \mathrm{IU} / \mathrm{ml}$ for heparinase I (E.C. 4.2.2.7; from Flavobacterium heparinum; Seikagaku, Tokyo, Japan) and $0.04 \mathrm{IU} / \mathrm{ml}$ for chondroitinase ABC (E.C. 4.2.2.4; from Proteus vulgaris; Sigma). Heparinase I cleaves the linkages GlcNS $\pm 6 \mathrm{~S}-\mathrm{IdoA} 2 \mathrm{~S}$ predominantly present in heparin and to a minor extent in HS [11]. Chondroitinase $\mathrm{ABC}$ cleaves CS and DS [11]. The solutions were incubated for $16 \mathrm{~h}$ at $37^{\circ} \mathrm{C}$.

Determination of glycosaminoglycan size

by polyacrylamide gel electrophoresis

Heparinoids and their enzymatic digestion products were separated by polyacrylamide gel electrophoresis using a $33 \%$ gel [36], fixed in $0.8 \%(w / v)$ alcian blue in $2 \%(v / v)$ HAc, and stained by combined azure A-silver treatment [38].

Production of anti-heparin antibodies

Antibodies (Table 2) were obtained by biopanning against HS or heparin, using bovine kidney HS (antibodies HS4C3 [41], HS3A8, HS4E4 [8], and LKIV69 [43]), porcine intestinal mucosa heparin (antibodies EW3D10 and EW4G2 [39]), human lung HS (antibody NS4F5; Smits et al., unpublished results), and mouse and human skeletal muscle GAG (antibodies AO4B08 and RB4EA12 [15], respectively). Large scale production of soluble antibodies was performed as described before $[8,41]$.

Enzyme-linked immunosorbent assay

Reactivity of the anti-heparin antibodies with the heparinoids was evaluated by enzyme-linked immunosorbent assay (ELISA). Wells of microtiter plates were coated overnight by incubation with the heparinoids that bound by passive adsorption [41]. After blocking with PBS containing
$3 \%(w / v)$ BSA and $1 \%(v / v)$ Tween-20 for $1 \mathrm{~h}$, anti-heparin antibodies [in PBS containing 1\% $(w / v)$ BSA and $0.1 \%(v / v)$ Tween-20] were added for $1.5 \mathrm{~h}$. As a negative control, antibody MPB49 was used, which is $>95 \%$ identical to most antibodies used, but does not bind any GAG. Bound antibodies were detected using mouse IgG anti-VSV tag antibody P5D4 (1:10; Boehringer Mannheim, Mannheim, Germany), followed by incubation with alkaline phosphataseconjugated rabbit anti-mouse IgG (1:2,000; Dako, Glostrup, Denmark), both for $1 \mathrm{~h}$. Enzyme activity was detected using $100 \mu 11 \mathrm{mg} p$-nitrophenyl phosphate (ICN, Aurora, $\mathrm{OH}$ ) $/ \mathrm{ml}$ $1 \mathrm{M}$ diethanolamine $/ 0.5 \mathrm{mM} \mathrm{MgCl}_{2}(\mathrm{pH} 9.8)$ as a substrate. Absorbance was measured at $405 \mathrm{~nm}$ [43]. Background reactivity, measured with the control antibody MPB49, was substracted. The absorbance with control antibody MPB49 was comparable to the absorbance measured when primary antibody was omitted.

Immunofluorescence staining

Human kidney cryosections $(2 \mu \mathrm{m})$ were incubated with anti-heparin antibodies in the absence or presence of $100 \mu \mathrm{g} / \mathrm{ml}$ of the heparinoids. Bound antibodies were detected as described [43].

\section{Results}

General biochemical analysis of heparinoids

The different classes of GAGs in the heparinoids were separated by agarose gel electrophoresis (Fig. 1). Heparin showed a band at about the same position as the HS standard, whereas the LMWHs dalteparin, nadroparin, enoxaparin, and tinzaparin migrated somewhat further into the gel. Danaparoid displayed bands at the level of HS and DS, whereas the CS band, which only represents $4 \%$ of the prepration (Table 1), could not be detected. Sulodexide clearly showed two bands, representing the 'fast-moving' heparin (heparin that is less sulfated and has a lower molecular weight [42]) and DS fractions. The chemically synthesized pentasaccharide fondaparinux, which has a much lower molecular weight compared to the other heparinoids, migrated further into the gel.

To analyze the molecular range of the heparinoids, gel electrophoresis using 33\% polyacrylamide was applied. The LMWHs clearly displayed distinct fragments with a lower molecular weight compared to heparin (Fig. 2a). The smaller fragments of enoxaparin compared to tinzaparin (both prepared by $\beta$-elimination) can be explained by a preference of heparinase for 2-O-sulfated iduronic acid in contrast to chemical alkaline treatment, which results in cleavage at both sulfated and non-sulfated iduronic acid 
Table 2 Characteristics of epitope-specific anti-heparin antibodies

\begin{tabular}{|c|c|c|c|c|c|c|}
\hline Antibody & $\begin{array}{l}\mathrm{V}_{\mathrm{H}} \mathrm{CDR} 3 \\
\text { sequence }\end{array}$ & $\begin{array}{l}\mathrm{V}_{\mathrm{H}} \\
\text { family }\end{array}$ & $\begin{array}{l}\text { DP } \\
\text { gene }\end{array}$ & $\begin{array}{l}\text { GAG used for } \\
\text { selection }\end{array}$ & Essential modifications & $\begin{array}{l}\text { Inhibitory } \\
\text { modifications }\end{array}$ \\
\hline HS4C3 & GRRLKD & 3 & 38 & Bovine kidney HS [41] & $\begin{array}{l}\mathrm{N} \text {-sulfation } \\
\text { 2-O-sulfation (minor } \\
\text { importance) } \\
\text { 6-O-sulfation } \\
\text { 3-O-sulfation }\end{array}$ & \\
\hline HS3A8 & GMRPRL & 3 & 38 & Bovine kidney HS [8] & $\begin{array}{l}\mathrm{N} \text {-sulfation } \\
\mathrm{C} 5 \text {-epimerization } \\
\text { 2-O-sulfation } \\
\text { 6-O-sulfation (likely) }\end{array}$ & \\
\hline HS4E4 & HAPLRNTRTNT & 3 & 38 & Bovine kidney HS [8] & $\begin{array}{l}\mathrm{N} \text {-acetylation } \\
\mathrm{N} \text {-sulfation } \\
\text { C5-epimerization }\end{array}$ & $\begin{array}{l}\text { 2-O-sulfation } \\
\text { 6-O-sulfation }\end{array}$ \\
\hline LKIV69 & GSRSSR & 3 & 38 & Bovine kidney HS [43] & $\begin{array}{l}\mathrm{N} \text {-sulfation } \\
\mathrm{C} 5 \text {-epimerization (likely) } \\
\text { 2-O-sulfation }\end{array}$ & 6-O-sulfation \\
\hline EW3D10 & GRTVGRN & 3 & 38 & $\begin{array}{l}\text { Porcine intestinal mucosa } \\
\text { heparin [39] }\end{array}$ & $\begin{array}{l}\text { Sulfation required, position(s) } \\
\text { unknown }^{\text {b }}\end{array}$ & \\
\hline EW4G2 & GKVKLPN & 3 & 38 & $\begin{array}{l}\text { Porcine intestinal mucosa } \\
\text { heparin [39] }\end{array}$ & $\begin{array}{l}\text { Sulfation required, position(s) } \\
\text { unknown }^{\text {b }}\end{array}$ & \\
\hline NS4F5 & SGRKGRMR & 3 & 53 & Human lung $\mathrm{HS}^{\mathrm{c}}$ & $\begin{array}{l}N \text {-sulfation } \\
\mathrm{C} 5 \text {-epimerization } \\
\text { 2-O-sulfation (high) } \\
6-O \text {-sulfation (high) }\end{array}$ & \\
\hline AO4B08 & SLRMNGWRAHQ & 3 & 47 & $\begin{array}{l}\text { Mouse skeletal muscle } \\
\text { GAG [15] }\end{array}$ & $\begin{array}{l}N \text {-sulfation } \\
\text { C5-epimerization } \\
\text { 2-O-sulfation }{ }^{\mathrm{d}} \\
\text { 6-O-sulfation (high) }\end{array}$ & \\
\hline RB4EA12 & RRYALDY & 3 & 32 & $\begin{array}{l}\text { Human skeletal muscle } \\
\text { GAG [15] }\end{array}$ & $\begin{array}{l}\mathrm{N} \text {-acetylation } \\
\mathrm{N} \text {-sulfation } \\
6 \text { - } \mathrm{O} \text {-sulfation }\end{array}$ & \\
\hline $\begin{array}{l}\text { MPB49 } \\
\text { (control) }\end{array}$ & WRNDRQ & 3 & 38 & - & - & \\
\hline
\end{tabular}

Given are the antibody name, amino acid sequence of the $\mathrm{V}_{\mathrm{H}}$ complementary determining region 3 (CDR3), $\mathrm{V}_{\mathrm{H}}$ germ line gene family, DP gene number, selection moiety, and relevant modification reactions required for antibody binding. Table derived from Wijnhoven et al. unpublished results.

GAG Glycosaminoglycan, $H S$ heparan sulfate

${ }^{a}$ Antibody HS4E4 possibly requires as yet unspecified $O$-sulfated residues [16].

${ }^{b}$ Although the chemical nature of the specific heparin/HS structure recognized is not exactly known, the antibody defines a unique sulfated heparin/HS structure as demonstrated by its specific staining pattern on renal cryosections $[8,39]$.

${ }^{\mathrm{c}}$ Smits et al., unpublished results

${ }^{\mathrm{d}}$ Antibody AO4B08 requires an internal 2-O-sulfated iduronic acid residue [16].

residues. Sulodexide and danaparoid showed a broad smear with less distinct fragments, in line with their methods of preparation, which does not include a specific depolymerization step. The pentasaccharide fondaparinux demonstrated primarily one individual band. To further establish the nature of the heparinoids, they were subjected to enzymatic digestion using heparinase I and/or chondroitinase $\mathrm{ABC}$ (Fig. 2b for dalteparin, enoxaparin, and sulodexide). Heparin, dalteparin, nadroparin, enoxaparin, tinzaparin, and fondaparinux were only cleaved by heparinase I, in accordance with their derivation from heparin. Sulodexide was cleaved by heparinase I as well as chondroitinase $A B C$, which is in line with the heparin/DS nature of the preparation. Danaparoid was primarily cleaved by chondroitinase $\mathrm{ABC}$, reflecting the presence of $\mathrm{DS} / \mathrm{CS}$, whereas heparinase I was less effective.

Characterization of heparinoids using anti-heparin antibodies

Reactivity of anti-heparin antibodies with immobilized heparinoids was analyzed by ELISA (Table 3). For 


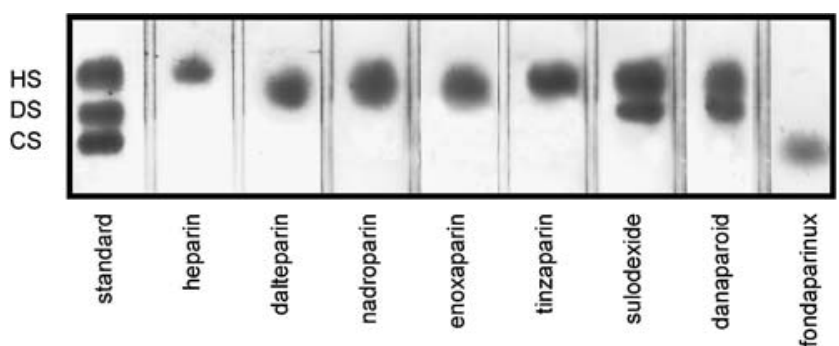

Fig. 1 Agarose gel electrophoresis of heparinoids. The different classes of GAGs in the drugs were visualized by a combined azure A-silver staining procedure. The standard contains $40 \mathrm{ng} \mathrm{HS}, 20 \mathrm{ng}$ DS, and $20 \mathrm{ng}$ CS

reference, reactivity of antibodies with heparin was set at $100 \%$. A distinct antibody-binding profile could be observed for all antibodies, although also similar reactivity with a number of antibodies was noticed. For instance, enoxaparin and tinzaparin, both prepared by $\beta$-elimination, reacted differently with antibodies LKIV69 and EW4G2, whereas they reacted similarly with the other antibodies. Nadroparin reacted very poorly with all the antibodies tested, which may be in line with its overall low amount of sulfated disaccharides in comparison with other LMWHs [17]. In contrast, sulodexide and danaparoid reacted strongly with antibody HS4E4, which defines a low-sulfated and $N$-acetylated

a

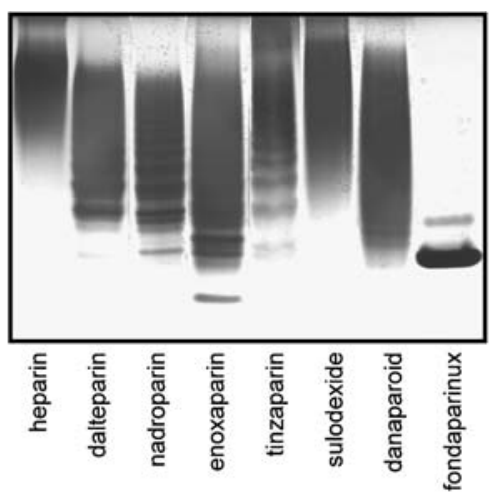

b

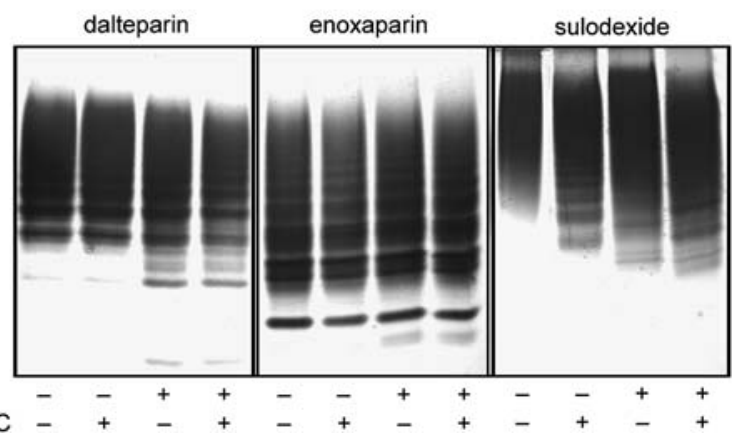

hep

Fig. 2 Thirty-three percent polyacrylamide gel electrophoresis of undigested (a) and enzymatically digested (b) heparinoids. Heparinoids were visualized by a combined alcian blue-silver staining procedure. Treatment with heparinase I resulted in cleavage of dalteparin and enoxaparin, whereas treatment with chondroitinase ABC did not. Sulodexide was cleaved by heparinase I as well as chondroitinase $\mathrm{ABC}$ epitope, in comparison to the LMWHs. This is in line with the presence of low-sulfated heparin (HS) and HS in sulodexide and danaparoid, respectively, and which contain a substantial amount of $N$-acetylated glucosamine. Compared to heparin, the other heparinoids reacted poorly with antibody NS4F5, which defines a very highly sulfated oligosaccharide.

Heparinoids could be ranked according to their reactivity with antibodies. For instance, reactivity of antibody EW4G2 was highest with heparin and sulodexide, followed by tinzaparin, dalteparin, and enoxaparin. Reactivity with the other heparinoids was very low or absent. Antibody LKIV69 reacted strongly with heparin, followed by sulodexide and tinzaparin, whereas other heparinoids reacted poorly. Reactivity of antibody AO4B08 was highest with heparin and sulodexide, followed by danaparoid and enoxaparin/tinzaparin/dalteparin, whereas reactivity with nadroparin was absent. These data indicate that distinct heparin domain structures are present in different commercially available heparinoids.

The only chemically defined heparinoid studied was the pentasaccharide fondaparinux (GlcNS(6OS)-GlcA-GlcNS $(3,6 \mathrm{O} S)-\operatorname{IdoA}(2 \mathrm{O} S)-\mathrm{GlcN} S(6 \mathrm{OS}))$. Due to its small size $(1,728 \mathrm{kDa})$, it was assayed using a competition ELISA (Table 4). Heparin was included as a reference and values obtained for fondaparinux were related to those obtained for heparin. Antibodies HS4E4 and RB4EA12, both requiring $\mathrm{N}$-acetylation in their epitopes, did not react with fondaparinux. Antibody NS4F5, which reacts with an extremely high sulfated oligosaccharide, was also not reactive. These results are in line with the chemical composition of fondaparinux, which contains no $\mathrm{N}$ acetylated glucosamine and a non-sulfated glucuronic acid residue. Reactivity with antibody LKIV69, which reacts poorly with 6-O-sulfated epitopes, was very weak and reflects the high amount of 6-O-sulfation in fondaparinux. Reactivity with antibody AO4B08, which needs C5epimerization, $\mathrm{N}$-sulfation, high $6-\mathrm{O}$-sulfation and an internal 2-O-sulfated iduronic acid residue, was only moderate/poor, reflecting the lack of an internal 2-Osulfated iduronic acid (i.e. flanked by other iduronic acid residues). Reactivity with antibody $\mathrm{HS} 4 \mathrm{C} 3$, which requires $\mathrm{N}-, 3-\mathrm{O}-$, and 6-O-sulfation, and antibody HS3A8, which requires at least $\mathrm{C} 5$-epimerization and $\mathrm{N}$ - and 2-Osulfation, was high, which corresponds with the chemical structure of fondaparinux. Reactivity was also high with antibodies EW3D10 and EW4G2, both defining an as yet undefined, but sulfated oligosaccharide. These data indicate that reactivity with antibodies may provide some chemical information of heparinoids.

Reactivity of the anti-heparin antibodies with immobilized heparinoids was supported by immunofluorescence analysis (Fig. 3). When human renal cryosections were 
Table 3 Reactivity of anti-heparin antibodies with heparinoids (ELISA)

\begin{tabular}{llllllllll}
\hline & HS4C3 & HS3A8 & HS4E4 & LKIV69 & EW3D10 & EW4G2 & NS4F5 & AO4B08 & RB4EA12 \\
\hline Heparin & 100 & 100 & 100 & 100 & 100 & 100 & 100 & 100 \\
Dalteparin & $75 \pm 6$ & $44 \pm 4$ & $8 \pm 5$ & $3 \pm 1$ & $33 \pm 6$ & $49 \pm 8$ & $0 \pm 0$ & $16 \pm 4$ & 100 \\
Nadroparin & $29 \pm 3$ & $5 \pm 2$ & $4 \pm 3$ & $0 \pm 0$ & $3 \pm 1$ & $7 \pm 4$ & $0 \pm 0$ & $1 \pm 1$ \\
Enoxaparin & $80 \pm 9$ & $56 \pm 8$ & $7 \pm 3$ & $10 \pm 2$ & $36 \pm 8$ & $26 \pm 7$ & $1 \pm 1$ & $28 \pm 7$ \\
Tinzaparin & $84 \pm 1$ & $55 \pm 2$ & $8 \pm 3$ & $44 \pm 10$ & $45 \pm 12$ & $74 \pm 1$ & $9 \pm 2$ & $20 \pm 3$ \\
Sulodexide & $102 \pm 5$ & $71 \pm 11$ & $283 \pm 21$ & $51 \pm 7$ & $43 \pm 10$ & $100 \pm 3$ & $0 \pm 0$ & $102 \pm 15$ & $26 \pm 8$ \\
Danaparoid & $69 \pm 11$ & $22 \pm 3$ & $58 \pm 17$ & $2 \pm 2$ & $2 \pm 1$ & $1 \pm 1$ & $0 \pm 0$ & $63 \pm 6$ & $3 \pm 1$ \\
\hline
\end{tabular}

Anti-heparin antibodies were applied to heparinoids immobilized on microtiter plates. Given is the reactivity of the antibodies with the heparinoids relative to unmodified heparin. Values are given as mean \pm SEM $(n=4)$. Each hepainoid shows a distinct immunoprofile.

simultaneously incubated with anti-heparin antibodies and heparinoids, staining was completely abolished with those heparinoids, which reacted strongly with the antibodies in ELISA (e.g. heparin and sulodexide for antibodies HS4C3 and HS4E4). An obvious decreased staining was noticed for those heparinoids, which reacted moderately with the antibodies (e.g. nadroparin for antibody HS4C3), whereas no major differences in staining were observed for those heparinoids, which did not bind to antibodies (e.g. nadroparin for antibody HS4E4).

\section{Discussion}

In this study eight heparinoids were characterized by their reactivity with nine different anti-heparin antibodies. All heparinoids showed a distinct antibody-binding profile in line with differences in GAG composition (heparin, HS, CS, DS), molecular weight, and method of preparation. A number of antibodies reacted strongly with certain heparinoids, but not with others. It may therefore be possible to deduce chemical and biological information of a specific heparinoid by virtue of its antibody profile. For instance, antibody NS4F5 showed reactivity with only heparin. This antibody defines a stretch of trisulfated $(\mathrm{N}-, 2-\mathrm{O}-$, and 6-Osulfated) and epimerized disaccharides and therefore defines a highly sulfated heparin domain (Smits et al., unpublished results). The lack of reactivity of the other heparinoids for NS4F5 indicates the absence or the destruction of the domain during their preparation. Absence may hold for sulodexide and danaparoid, which contain HS rather than heparin. Destruction by the depolymerization processes may hold for the LMWHs. In this respect, reactivity with antibody NS4F5 may be indicative for the degree of depolymerization. The antibody profile of the chemically synthesized pentasaccharide fondaparinux is compatible with its chemical structure GlcNS(6OS)GlcA-GlcNS(3,6OS)-IdoA(2OS)-GlcNS(6OS). Reactivity with antibodies HS4C3 and HS3A8 indicates it to contain iduronic acid and $\mathrm{N}-, 2-\mathrm{O}-, 3-\mathrm{O}-$, and 6-O-sulfate residues, whereas poor/no reactivity with antibodies HS4E4, LKIV69, AO4B08, and RB4EA12 indicates the lack of internal 2- $O$-sulfated iduronic acid residues (i.e. flanked by other iduronic acids) and $N$-acetylated glucosamine residues. Lack of reactivity with antibody NS4F5 indicates that fondaparinux does not contain a stretch of tri-sulfated disaccharides.

Next to information on the chemical properties of the heparinoids, the antibody profile may be indicative for their biological activity, e.g. the anti-Xa/anti-IIa ratio. This ratio is of essential importance since it is associated with a more predictable anticoagulant effect. The anti-Xa/anti-IIa ratio is 1 for unfractionated heparin, 2-4 for LMWHs, 0.5 for sulodexide, and infinite for fondaparinux [2, 23]. Antibodies such as HS4E4 and AO4B08 (Tables 3 and 4), which are highly reactive with heparin and sulodexide, but poorly/not with LMWHs and fondaparinux, likely bind to a heparin domain structure at a site proximal to the

Table 4 Reactivity of anti-heparin antibodies with heparin and fondaparinux (competition ELISA)

\begin{tabular}{llllllllll}
\hline & HS4C3 & HS3A8 & HS4E4 & LKIV69 & EW3D10 & EW4G2 & NS4F5 & AO4B08 & RB4EA12 \\
\hline Heparin & $0.3 \pm 0.1$ & $0.4 \pm 0.1$ & $2.7 \pm 0.1$ & $0.2 \pm 0.0$ & $0.1 \pm 0.0$ & $0.1 \pm 0.0$ & $0.1 \pm 0.0$ & $1.2 \pm 0.2$ & $<0.01$ \\
Fondaparinux & $1.7 \pm 0.2$ & $1.3 \pm 0.1$ & $>200$ & $10.3 \pm 0.8$ & $0.2 \pm 0.0$ & $1.5 \pm 0.5$ & $11.7 \pm 0.8$ & $12.3 \pm 3.8$ & $21.5 \pm 0.5$ \\
Ratio fondaparinux/heparin & 5.7 & 3.3 & $>74$ & 51.5 & 2 & 15 & 117 & 10.3 & $>2150$ \\
\hline
\end{tabular}

Given are the amounts of heparinoids (micrograms per milliliter), which result in a $50 \%$ inhibition of the binding of the anti-heparin antibodies to immobilized $\mathrm{HS} /$ heparin $\left(\mathrm{IC}_{50}\right.$ values), and the ratio between antibody reactivity with fondaparinux and heparin. Values are given as mean $\pm \mathrm{SEM}$ $(n=3)$. Antibody EW3D10 showed a strong reactivity with fondaparinux, followed by antibodies HS4C3, HS3A8, and EW4G2. The other antibodies showed a relatively weak or no reactivity. 

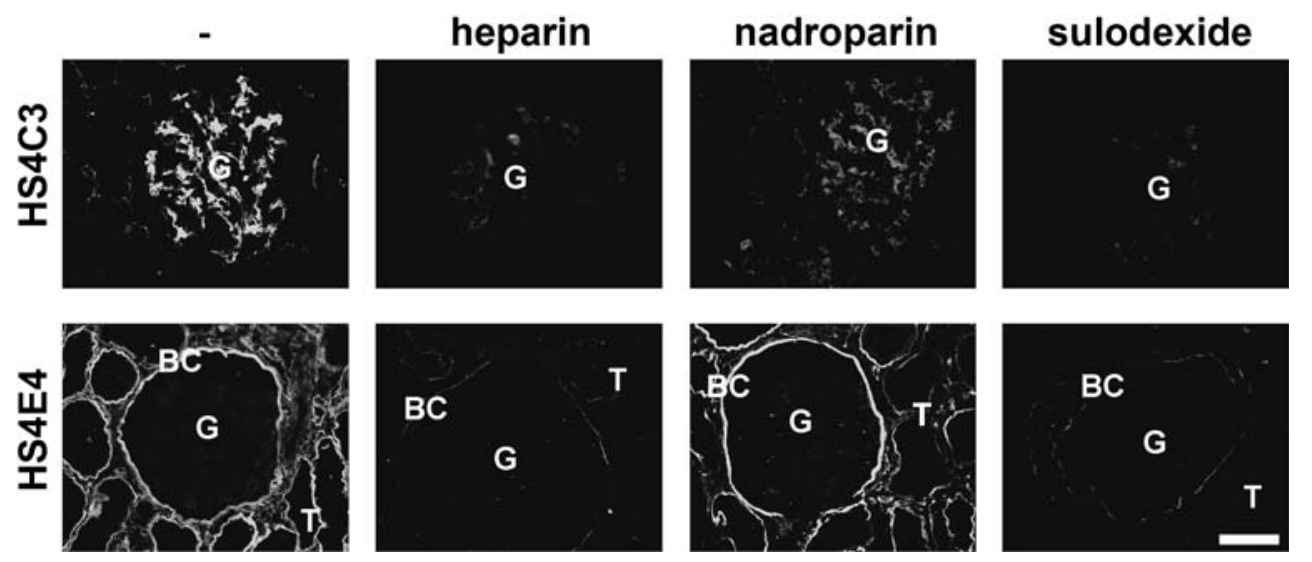

Fig. 3 Immunofluorescence staining of human renal cryosections with anti-heparin antibodies HS4C3 and HS4E4 in the absence and presence of heparinoids. Staining was abolished when renal cryosections were incubated with $\mathrm{HS} 4 \mathrm{C} 3$ or HS4E4 in the presence of heparin

pentasaccharide sequence and may be indicative for a high anti-factor IIa activity. These results indicate that when a heparinoid is recognized by antibodies HS4C3, HS3A8, EW3D10, and EW4G2 (which showed a high reactivity with fondaparinux; Table 4), as well as by antibodies HS4E4 and AO4B08, the heparinoid is able to form a ternary complex with antithrombin III and factor IIa, resulting in inactivation of both factors Xa and IIa [23]. On the other hand, when a heparinoid is recognized by antibodies HS4C3, HS3A8, EW3D10, and EW4G2, but not by antibodies HS4E4 and AO4B08, it is does not contain the heparin domain structure at the site proximal to the pentasaccharide required for factor IIa binding. In this case only factor Xa will be inactivated. When a ratio is calculated from reactivity of heparinoids with antibodies $\mathrm{HS} 4 \mathrm{C} 3$ and HS4E4 (indicative for 3-O-sulfates and for the presence of domains allowing IIa activity, respectively), and heparin is assigned a ratio of 1 , the following numbers are found: dalteparin, 9; nadroparin, 7; enoxaparin, 11; tinzaparin, 11; sulodexide, 0.4; and fondaparinux, >>13 (no antibody HS4E4 reactivity observed). These numbers are in line with the above mentioned anti-Xa/anti-IIa ratios, which were determined by enzymatic assays [2, 23], and indicate that biological characteristics are associated with antibody profiles.

Heparin-induced thrombocytopenia (HIT) is a major complication in patients administered heparin. Heparin binding to platelet factor 4 (PF4) causes a conformational change in the protein, rendering it antigenic. Susceptible patients develop antibodies to the heparin-PF4 complex, and binding of the antibody-PF4-heparin complex to its receptor on platelets results in platelet activation and aggregation. In addition, platelets are removed by activated immune cells, resulting in thrombocytopenia [6]. Highly sulfated domains within heparin/HS are involved in the or sulodexide. HS4C3 staining was decreased in the presence of nadroparin, whereas HS4E4 staining was unaffected. Bar represents $50 \mu \mathrm{m}$; magnification is identical for each photograph. $G$ Glomerulus, $B C$ Bowman's capsule, $T$ renal tubule

binding to PF4 [31], and an increase in sulfation and size is associated with an increased risk of HIT [6, 19]. This may explain why the incidence of HIT is lower in patients treated with LMWHs, danaparoid, or fondaparinux compared to unfractionated heparin [7, 23]. Antibody NS4F5, which defines a highly sulfated domain, reacted strongly with heparin, but not with the other heparinoids. Reactivity with this antibody may thus be used to obtain information about the risk of a heparinoid to induce HIT.

Although we have focussed here on the immunoprofiling of heparinoids in the context of anticoagulation, this technique may also be useful to evaluate heparinoids in other settings. Heparin and LMWHs have been used to treat patients with kidney disease since an ameliorating effect on a.o. proteinuria has been found $[1,14,21,25,30,33,34$, 40]. Likewise, heparinoids are reported to have anti-tumor and anti-metastatic effects [3, 20, 29], the degree of sulfation being of crucial importance. Heparinase IIIdigested heparin (highly sulfated fragments), inhibited tumor growth, whereas heparinase I-digested heparin (low sulfated fragments) promoted tumor growth [18], underscoring the necessity to characterize the preparation used. In addition to the implementation of these antibodies to characterize the therapeutic potential of heparinoids, they may be used to stage a disease (e.g. kidney disease, cancer) characterized by a specific HS profile in tissue, blood, and/ or urine.

Since the antibody profile of the heparinoids used in these studies may be correlated to a specific biological effect, antibodies may be used for the identification and subsequent characterization of the active structures within the heparinoids, which may result in the development of more specific drugs. The antibodies described here may also be used to counteract bleeding in patients administered an overdose of heparinoids. This is generally accomplished 
by treatment with protamine sulfate $[9,26]$, but this rather unspecific polycation may cause hypotension, bradycardia, and thrombocytopenia. In addition it may cause other severe side effects including anaphylactic and anaphylactoid reactions, resulting in respiratory distress, circulatory collapse, capillary leak, and pulmonary hypertension [26]. There is a need for safer agents that inhibit the anticoagulant activity of heparinoids, and some antibodies described here may be useful, especially those that also react with fondaparinux, which is difficult to inhibit in vivo. It has already been described that antibodies HS4C3 and EW3D10 strongly inhibited heparin-induced anticoagulation (APTT clotting assay) [35, 39]. Antibody HS4C3 also blocked the anticoagulant activities of heparin and fondaparinux in an anti-factor Xa assay [35]. These studies indicate that anti-heparin antibodies may react with heparinoids thereby neutralizing their anticoagulant effect.

Finally, our antibodies may be used for fast industrial screening of heparinoids. A quality check of heparinoid batches may be performed using the antibodies since each heparinoid shows a specific immunoprofile.

In conclusion, this study presents a novel way of characterizing heparinoids using immunoprofiling. Reactivity with antibodies was found indicative for chemical and biological aspects of the heparinoids, and may therefore be used for a fast and simple screening of industrial batches for defined characteristics.

Acknowledgement This work was supported by grants from The Netherlands Organization for Scientific Research (NWO; program grant 902-27-292), the International Human Frontier Science Program Organization (HFSP; grant RGP0062/2004-C101), and the Dutch Kidney Foundation (grant C05.2152).

\section{References}

1. Achour A., Kacem M., Dibej K., Skhiri H., Bouraoui S., El May M.: One year course of oral sulodexide in the management of diabetic nephropathy. J. Nephrol. 18, 568-574 (2005)

2. Baglin T., Barrowcliffe T.W., Cohen A., Greaves M.: Guidelines on the use and monitoring of heparin. Br. J. Haematol. 133, 19-34 (2006)

3. Bobek V., Kovarik J.: Antitumor and antimetastatic effect of warfarin and heparins. Biomed. Pharmacother. 58, 213-219 (2004)

4. Casu B., Torri G.: Structural characterization of low molecular weight heparins. Semin. Thromb. Hemost. 25(Suppl 3), 17-25 (1999)

5. Cheng J.W.: Fondaparinux: a new antithrombotic agent. Clin. Ther. 24, 1757-1769 (2002)

6. Chong B.H.: Heparin-induced thrombocytopenia. J. Thromb. Hemost. 1, 1471-1478 (2003)

7. Chong B.H., Magnani H.N.: Orgaran in heparin-induced thrombocytopenia. Haemostasis. 22, 85-91 (1992)

8. Dennissen M.A., Jenniskens G.J., Pieffers M., Versteeg E.M., Petitou M., Veerkamp J.H., van Kuppevelt T.H.: Large, tissueregulated domain diversity of heparan sulfates demonstrated by phage display antibodies. J. Biol. Chem. 277, 10982-10986 (2002)

9. Dietrich C.P., Shinjo S.K., Moraes F.A., Castro R.A., Mendes A., Gouvea T.C., Nader H.B.: Structural features and bleeding activity of commercial low molecular weight heparins: neutralization by ATP and protamine. Semin. Thromb. Hemost. 25(Suppl 3), 43-50 (1999)

10. Dunn C.J., Sorkin E.M.: Dalteparin sodium. A review of its pharmacology and clinical use in the prevention and treatment of thromboembolic disorders. Drugs 52, 276-305 (1996)

11. Ernst S., Langer R., Cooney C.L., Sasisekharan R.: Enzymatic degradation of glycosaminoglycans. Crit. Rev. Biochem. Mol. Biol. 30, 387-444 (1995)

12. Fareed J., Leong W.L., Hoppensteadt D.A., Jeske W.P., Walenga J., Wahi R., Bick R.L.: Generic low-molecular-weight heparins: some practical considerations. Semin. Thromb. Hemost. 30, 703-713 (2004)

13. Farndale R.W., Buttle D.J., Barrett A.J.: Improved quantitation and discrimination of sulphated glycosaminoglycans by use of dimethylmethylene blue. Biochim. Biophys. Acta. 883, 173-177 (1986)

14. Gambaro G., Kinalska I., Oksa A., Pont'uch P., Hertlova M., Olsovsky J., Manitius J., Fedele D., Czekalski S., Perusicova J., Skrha J., Taton J., Grzeszczak W., Crepaldi G.: Oral sulodexide reduces albuminuria in microalbuminuric and macroalbuminuric type 1 and type 2 diabetic patients: the Di.N.A.S. randomized trial. J. Am. Soc. Nephrol. 13, 1615-1625 (2002)

15. Jenniskens G.J., Oosterhof A., Brandwijk R., Veerkamp J.H., van Kuppevelt T.H.: Heparan sulfate heterogeneity in skeletal muscle basal lamina: demonstration by phage display-derived antibodies. J. Neurosci. 20, 4099-4111 (2000)

16. Kurup S., Wijnhoven T.J., Jenniskens G.J., Kimata K., Habuchi H., Li J.P., Lindahl U., van Kuppevelt T.H., Spillmann D.: Characterization of anti-heparan sulfate phage-display antibodies AO4B08 and HS4E4. J. Biol. Chem. 282(29), 21032-21042 (2007)

17. Linhardt R.J., Gunay N.S.: Production and chemical processing of low molecular weight heparins. Semin. Thromb. Hemost. 25 (Suppl 3), 5-16 (1999)

18. Liu D., Shriver Z., Venkataraman G., El Shabrawi Y., Sasisekharan R.: Tumor cell surface heparan sulfate as cryptic promoters or inhibitors of tumor growth and metastasis. Proc. Natl. Acad. Sci. U.S.A. 99, 568-573 (2002)

19. Maccarana M., Lindahl U.: Mode of interaction between platelet factor 4 and heparin. Glycobiology 3, 271-277 (1993)

20. Mousa S.A.: Anticoagulants in thrombosis and cancer: the missing link. Semin. Thromb. Hemost. 28, 45-52 (2002)

21. Myrup B., Hansen P.M., Jensen T., Kofoed-Enevoldsen A., FeldtRasmussen B., Gram J., Kluft C., Jespersen J., Deckert T.: Effect of low-dose heparin on urinary albumin excretion in insulindependent diabetes mellitus. Lancet 345, 421-422 (1995)

22. Neely J.L., Carlson S.S., Lenhart S.E.: Tinzaparin sodium: a lowmolecular-weight heparin. Am. J. Health-Syst. Pharm. 59, 1426-1436 (2002)

23. Petitou M., van Boeckel C.A.: A synthetic antithrombin III binding pentasaccharide is now a drug! What comes next? Angew. Chem. Int. Ed. Engl. 43, 3118-3133 (2004)

24. Polkinghorne K.R., McMahon L.P., Becker G.J.: Pharmacokinetic studies of dalteparin (Fragmin), enoxaparin (Clexane), and danaparoid sodium (Orgaran) in stable chronic hemodialysis patients. Am. J. Kidney Dis. 40, 990-995 (2002)

25. Poplawska A., Szelachowska M., Topolska J., Wysocka-Solowie B., Kinalska I.: Effect of glycosaminoglycans on urinary albumin excretion in insulin-dependent diabetic patients with micro- or macroalbuminuria. Diabetes Res. Clin. Pract. 38, 109-114 (1997)

26. Porsche R., Brenner Z.R.: Allergy to protamine sulfate. Heart Lung. 28, 418-428 (1999) 
27. Prydz K., Dalen K.T.: Synthesis and sorting of proteoglycans. J. Cell Sci. 113(Pt 2), 193-205 (2000)

28. Sagedal S., Hartmann A., Sundstrom K., Bjornsen S., Fauchald P., Brosstad F.: A single dose of dalteparin effectively prevents clotting during haemodialysis. Nephrol. Dial. Transplant. 14, 1943-1947 (1999)

29. Sasisekharan R., Shriver Z., Venkataraman G., Narayanasami U.: Roles of heparan-sulphate glycosaminoglycans in cancer. Nat. Rev. Cancer 2, 521-528 (2002)

30. Skrha J., Perusicova J., Pont'uch P., Oksa A.: Glycosaminoglycan sulodexide decreases albuminuria in diabetic patients. Diabetes Res. Clin. Pract. 38, 25-31 (1997)

31. Stringer S.E., Gallagher J.T.: Specific binding of the chemokine platelet factor 4 to heparan sulfate. J. Biol. Chem. 272, 20508-20514 (1997)

32. Sugahara K., Kitagawa H.: Recent advances in the study of the biosynthesis and functions of sulfated glycosaminoglycans. Curr. Opin. Struct. Biol. 10, 518-527 (2000)

33. Szelachowska M., Poplawska A., Topolska J., Kinalska I., Grimaldi M., Szelanowska M., Jopdska J.: A pilot study of the effect of the glycosaminoglycan sulodexide on microalbuminuria in type I diabetic patients. Curr. Med. Res. Opin. 13, 539-545 (1997)

34. Tamsma J.T., van der Woude F.J., Lemkes H.H.: Effect of sulphated glycosaminoglycans on albuminuria in patients with overt diabetic (type 1) nephropathy. Nephrol. Dial. Transplant. 11, 182-185 (1996)

35. Ten Dam G.B., Kurup S., van de Westerlo E.M., Versteeg E.M., Lindahl U., Spillmann D., van Kuppevelt T.H.: 3-O-sulfated oligosaccharide structures are recognized by anti-heparan sulfate antibody HS4C3. J. Biol. Chem. 281, 4654-4662 (2006)
36. Turnbull J.E., Hopwood J.J., Gallagher J.T.: A strategy for rapid sequencing of heparan sulfate and heparin saccharides. Proc. Natl. Acad. Sci. U.S.A. 96, 2698-2703 (1999)

37. Turpie A.G.: Pentasaccharides. Semin. Hematol. 39, 158-171 (2002)

38. van de Lest C.H., Versteeg E.M., Veerkamp J.H., van Kuppevelt T.H.: Quantification and characterization of glycosaminoglycans at the nanogram level by a combined azure A-silver staining in agarose gels. Anal. Biochem. 221, 356-361 (1994)

39. van de Westerlo E.M., Smetsers T.F., Dennissen M.A., Linhardt R.J., Veerkamp J.H., van Muijen G.N., van Kuppevelt T.H.: Human single chain antibodies against heparin: selection, characterization, and effect on coagulation. Blood 99, 2427-2433 (2002)

40. van der Pijl J.W., van der Woude F.J., Geelhoed-Duijvestijn P.H., Frolich M., van der Meer F.J., Lemkes H.H., van Es L.A.: Danaparoid sodium lowers proteinuria in diabetic nephropathy. J. Am. Soc. Nephrol. 8, 456-462 (1997)

41. van Kuppevelt T.H., Dennissen M.A., van Venrooij W.J., Hoet R. M., Veerkamp J.H.: Generation and application of type-specific anti-heparan sulfate antibodies using phage display technology. Further evidence for heparan sulfate heterogeneity in the kidney. J. Biol, Chem, 273, 12960-12966 (1998)

42. Volpi N., Cusmano M., Venturelli T.: Qualitative and quantitative studies of heparin and chondroitin sulfates in normal human plasma. Biochim. Biophys. Acta. 1243, 49-58 (1995)

43. Wijnhoven T.J., Lensen J.F., Rops A.L., van der Vlag J., Kolset S. O., Bangstad H.J., Pfeffer P., van den Hoven M.J., Berden J.H., van den Heuvel L.P., van Kuppevelt T.H.: Aberrant heparan sulfate profile in the human diabetic kidney offers new clues for therapeutic glycomimetics. Am. J. Kidney Dis. 48, 250-261 (2006) 